\title{
QUO VADIS QUALITÄTSENTWICKLUNG? ÜBER STRATEGIEN, STRUKTUREN UND INSTRUMENTEN VON HEUTE UND MORGEN.
}

\author{
ATTILA PAUSITS \\ DOI: $10.22163 /$ fteval.2019.460
}

$\mathrm{E}$ ine der wesentlichen Säulen der Qualitätssicherung ist die Evaluation im Hochschulwesen. Diese durchdringt alle relevanten und möglichen Bereiche eines Hochschulsystems und kommt mit dem Modell des Peer-Reviews einem akademischen Miteinander nahe. Es werden Systeme, Hochschulen, einzelne Fakultäten, wissenschaftliche oder administrative Organisationseinheiten und gar die Leistungen einzelner Personen gemessen, bewertet und Empfehlungen für Verbesserungen verschriftlicht und ausgesprochen. Die verwendete Sprache ist meistens konstruktiv, fundiert und so in wissenschaftlichen Traditionen verwurzelt. Dies vermittelt sowohl den Beteiligten als auch den Betroffenen eine gewisse Sicherheit. Um das Qualitätskulturmodell der Bewertung und Weiterentwicklung, die ihre Wurzeln im New Public Management und Neoinstitutionalismus hat und durch ein modernes Rechenschaftsprinzip durch die Hochschulpolitik angetrieben wird, implementieren zu können, braucht es eine große Anzahl an Personen, die als Reviewer und Reviewerinnen diese Prozesse mit ihrer Fachexpertise stützen.

Zugegebenermaßen kommen wir immer weiter weg von Modellen der Akkreditierung von (Studien)Programmen, die z.B. in den letzten Jahren in Litauen und in anderen Ländern eine wahre Völkerwanderung von internationalen Expertinnen und Experten und eine noch nie dagewesene Qualitätssicherungswelle ausgelöst haben. Programmakkreditierungen sind nicht nur sehr ressourcenintensiv, sondern bei einem intakten Qualitätsmanagementsystem der Institutionen auch nicht zwingend notwendig. Sie wurden gerade bei der Einführung der Qualitätssicherung in vielen Hochschulsystemen verbreitet verwendet - oft international besetzt um möglichen Verflechtungen und Einflüssen in relativ kleinen Hochschulsystemen entgegenzuwirken. Damit wollte man sich dem „Academic Inbreeding der Qualitätssicherung" entgegenstellen und für eine objektivere Bewertung sorgen. Solche Evaluationen benötigen aber mehr als nur die Expertise in einem bestimmten Fachgebiet. Vielmehr geht es um komplexes Wissen, welches auf einem soliden Systemverständnis basiert. Wie ist das nationale Hochschulsystem strukturiert und finanziert? Welche Rahmenbedingungen finden sich für das Handeln im Hochschulbereich? Was für Bewertungskriterien existieren für Professorinnen und Professoren? Welche Governance-Instrumente werden eingesetzt? Es ist offensichtlich, dass viele Aspekte auf die Einbettung und Kontextualisierung von Qualität in einem bestimmten Land eine Wirkung erzielen. Daher ist es umso wichtiger, Qualität relational und kontextual zu messen und zu bewerten. Dieser Anspruch ist auch ein wesentlicher Kritikpunkt an internationalen Rankings. Ohne Kontext können die Peers lediglich nur allgemeingültige Faktoren als Referenz verwenden und die Rückmeldungen und Empfehlungen gehen dann von einem minimalistischen Verständnis aus. Trotz eines gemeinsamen europäischen Hochschulraums, Harmonisierungsinitiativen wie der Bologna Prozess oder gemeinsamen Qualitätsmodellen wie die „Standards and Guidelines for Quality Assurance in the European Higher Education Area" (ESG) ist der Umgang mit nationalen, regionalen, lokalen und institutionellen Gegebenheit erforderlich. Dies verlangt eine rational aufgesetzte, fachlich geführte und einfühlsam gestaltete verantwortungsbewusste Evaluation. Internationale und gerade periodisch wiederkehrende Evaluationen können daher mehr Transparenz, eine Annäherung an internationale Qualitätsstandards und eine Verbesserung des eigenen Hochschulsystems durch die Betrachtung von außen leisten.

Insbesondere die kontinuierliche Verbesserung steht immer mehr im Mittelpunkt moderner Qualitätssicherungsmodelle. Der Begriff der Sicherung ist fast schon altmodisch und wird z.B. in politischen oder strategischen Dokumenten der Makro-, Meso- und Mikrobenen der Hochschulsteuerung immer mehr verbannt. So stellt man richtigerweise die Frage: Ist die Evaluation (noch) das richtige Tool, um Qualität zu verbessern? Oder versuchen wir doch die Zukunft, zum Ausdruck gebracht durch den Begriff „Entwicklung“, mit den Instrumenten der Vergangenheit zu bestimmen? Wären da nicht neue Methoden wie "prädiktive Analysen" bessere Methoden, um etwas in der Zukunft zu entwickeln? Fragen, die eine Evaluation der Evaluation begründen können, wie auch diese Publikation den Versuch unternimmt, unterschiedliche Perspektiven der Evaluation zusammenzuführen und mögliche Zukunftsszenarien zu skizzieren.

Zweifelsohne haben Hochschulsysteme in den letzten zwei Dekaden mit den neuen Instrumenten wie Evaluation und Peer-Review ausreichend Erfahrungen gesammelt. Nicht nur Systeme, sondern auch Hochschulen, die am laufenden Band solche Prozesse initiieren, weil sie (gesetzlich) vorgeschrieben sind, und umsetzen, haben sowohl Verfahrens-Know-How gesammelt als auch professionelle Organisationseinheiten der Qualitätsentwicklung aufgebaut. Dies sind sicherlich beachtliche Erfolge der Organisationsentwicklung. Heutzutage verfügen Hochschulen und Agenturen, die mit einer fachlichen Versiertheit ausgestattet sind, solche Prozesse unterstützen und dadurch die Ergebnisqualität der Verfahren entscheidend positiv mitbeeinflussen, über gut ausgebildete Qualitätsmanagerinnen und -manager. Die Hochschulen haben Strukturen geschaffen und diese werden durch standardisierte Prozesse gestützt. Von der Datensammlung, Selbstbericht und Besuche 
im Sinne eines "Informed Peer-Review" bis hin zum Umgang mit den Ergebnissen arbeiten Hochschulen effektiv und effizient entlang der (in den meisten Fällen von außen vorgegebenen) Prozesse. Trainings wie formale Abschlüsse eines weiterbildenden Hochschulmanagement Masters haben hier auch wesentliche Beiträge der Professionalisierung geleistet. Auch wenn in Europa solche Programme erst seit Anfang der 2000er Jahre angeboten werden, wie das in 2002 gestartete erste „MBA in Higher Education Management" am Institute of Education in London, das Hochschul- und Wissenschaftsmanagement MSc an der Hochschule in Osnabrück, oder an der Donau Universität Krems als einziges Programm hierzulande, haben diese auch zur Professionsentwicklung des Managements der Hochschulen und damit zur Qualitätsentwicklung generell wie auch zur Personalentwicklung beigetragen.

Die Verbesserung der Verfahren hängt nicht nur von den Hochschulangehörigen selbst, sondern auch von den Qualitätsagenturen ab. Gerade die Agenturen im Europäischen Hochschulraum haben sich von Systemanbieterinnen zu internationalen Playern entwickelt. Viele agieren nicht nur im eigenen Hochschulsystem, sondern bieten auch für Hochschulen in anderen Ländern Leistungen an. Auch die Vernetzung und der Austausch zwischen diesen Agenturen durch das „European Association for Quality Assurance in Higher Education" (ENOA) und internationale europäische Projekte, wie das „Sustainable Quality Enhancement in Higher Education Learning and Teaching" unterstützen die Entwicklung der Qualitätsentwicklungsmaßnahmen wie beispielsweise im oben genannten Projekt durch einen international vergleichenden Modellrahmen der Qualitätsentwicklung im Lehren und Lernen sowie ein umfassendes Indikatorenset. Diese und ähnliche Initiativen leisten auch einen wesentlichen Beitrag der theoretischen Fundierung und Weiterentwicklung gängiger Evaluierungen. Die Methoden und Prozesse der Hochschulevaluierung haben sich in den letzten Jahren durch die Prozesserfahrungen und die kritische Auseinandersetzung mit Hochschulevaluationen sowie das „Heranbilden“ von Peers, die solche Verfahren akademisch und/oder operativ leiten und einer noch größeren akademischen Gruppe an Mitgliedern von Peerverfahren, die regelmäßig an solchen Evaluationen für die unterschiedlichen Agenturen aber auch andere Fachorganisationen teilnehmen, ständig weiterentwickelt.

Die Hochschulforschung selbst hat den Forschungsbereich der Evaluationen ebenso für sich entdeckt. Es wird nicht nur regelmäßig dazu in facheinschlägigen Journals publiziert, sondern die Hochschulevaluation hat durch spezialisierte und wissenschaftlich anerkannte Fachzeitschriften wie "Quality in Higher Education" oder "Qualität in der Wissenschaft" für eine Akademisierung des Feldes gesorgt. Hier werden aber nicht nur klassische Beiträge der Hochschulforschung publiziert, sondern auch Fallstudien und Erfahrungsberichte, die eher dem Institutional Research zugeordnet werden können. Institutional Research erforscht u.a. die eigene Einrichtung, unterstützt strategische Entscheidungsprozesse und leistet einen wesentlichen Beitrag zur Weiterentwicklung der Hochschule. Das organisationale Wissen wird durch die Sammlung und Auswertung von hochschulinternen Daten gestützt und durch statistische Methoden evidenzbasiert angereichert. Eine strategisch ausgerichtete Qualitätsentwicklung, die nicht nur primär eine Legitimationsfunktion der Evaluation vorsieht, sondern vielmehr an Kapazitäten denkt, die nicht nur eine Ex-Post- sondern vielmehr eine Ex-Ante-Betrachtung präferiert und dadurch stärker auf die Zukunft ausgerichtet ist, benötigt andere Fähigkeiten und Fertigkeiten. Aktuell werden Evaluationen aus Ergebnissen und Daten der Vergangenheit gestützt. In der Zukunft wird es ebenso wichtig sein, die Kapazitäten, die über eine Qualitätssicherung hinausgehen, in den Evaluationen zu berücksichtigen. Hier wird es um Strukturen, Prozesse und Leistungen gehen, die strategische Entscheidungsprozesse evidenzbasiert unterstützen. Aufgaben und Leistungen, die zukünftige Qualitätsszenarien stärker in den Mittelpunkt rücken und so die Robustheit aber auch eine neue Ausrichtung des Qualitätsmanagements aufzeigen. Hier wird die Berücksichtigung und Einbindung von Institutional Research an Bedeutung zunehmen.

So wie das Institutional Research an Bedeutung für die Qualitätsentwicklung gewinnt, ist davon auszugehen, dass weitere Themen in die Evaluation einfließen werden. Neben Lehre und Forschung wird auch die „Dritte Mission”, wie die wissenschaftliche Weiterbildung oder Technologie- und Wissenstransfer in der Vergangenheit, mit weiteren Bereichen in die Evaluation einfließen. Soziales Engagement als substantieller Teil der hochschulischen Aufgabe gesellschaftlicher Verantwortung wird in den neuen Qualitätsmodellen genauso an Bedeutung zunehmen. Daher wird es wichtig sein, den Bereich der Dritten Mission sowohl mit Deskriptoren als auch Indikatoren in einem Qualitätsmodell einer Hochschule sichtbar zu machen. Hier stehen die existierenden Evaluationsmodelle erst am Anfang.

Bereits seit längerem werden auch in der Wirtschaft Audits durchgeführt, welche das Innovationsmanagement einer Organisation evaluieren. Dabei geht es um die Messung der Innovationskraft und die richtige Einschätzung von Potentialen wie z.B. von Forschungsergebnissen in Hinblick auf Marktfähigkeit. Diese Prozesse tragen dazu bei, Forschungsergebnisse zu Innovationen weiterzuentwickeln. In den letzten Jahren haben Hochschulen sehr viel in das Forschungsmanagement investiert und so Kernprozesse der Forschung durch Unterstützungsangebote ergänzt. Diese Prozesse sind bereits Gegenstand gängiger Evaluationsmodelle. In einem nächsten Schritt wird es gleichwohl wichtig sein, das Modell des Innovationsaudits in die Hochschulen einzuführen und das Portfolio der Qualitätssicherungsagenturen damit zu erweitern. Konzeptionelle Modelle existieren bereits. Nun müssen diese adaptiert und innerhalb des Qualitätsmanagement-Modells der Hochschulen auch beachtet werden.

Die bereits entwickelten modularen Evaluationsmodelle der Agenturen werden in der Zukunft mit weiteren Modulen und Möglichkeiten erweitert werden. Dabei wird es aber nicht darum gehen, diese allumfassend zu evaluieren. Vielmehr gilt es dann entlang von institutionellen Strategien, die Qualitätsentwicklung durch Evaluation oder mit Hilfe von Innovationsaudits zu unterstützen. Im Kern geht es nicht nur darum, dass eine Hochschule durch die gesetzlichen Vorgaben Mindeststandards erfüllt, sondern auch um strategische Maßnahmen, damit die Qualitätspotentiale z.B. durch eine bessere Positionierung ausgeschöpft werden können.

\section{AUTOR}

\section{ASS.PROF. DKFM. DR. HABIL ATTILA PAUSITS}

Leiter - Zentrum für Bildungsmanagement und Hochschulentwicklung, Donau-Universität Krems

Dr-Karl-Dorrek-Straße 30, 3500 Krems

E: attila.pausits@donau-uni.ac.at

https://orcid.org/0000-0003-2901-5575 